\title{
New properties of a certain method of summation of generalized hypergeometric series
}

\author{
Rafał Nowak* Paweł Woźny ${ }^{\dagger}$
}

August 30, 2016

\begin{abstract}
In a recent paper (Appl. Math. Comput. 215, 1622-1645, 2009), the authors proposed a method of summation of some slowly convergent series. The purpose of this note is to give more theoretical analysis for this transformation, including the convergence acceleration theorem in the case of summation of generalized hypergeometric series. Some new theoretical results and illustrative numerical examples are given.
\end{abstract}

\section{Introduction}

The generalized hypergeometric series ${ }_{p} \mathrm{~F}_{q}\left(\alpha_{1}, \ldots, \alpha_{p} ; \beta_{1}, \ldots, \beta_{q} \mid x\right)$ is defined by

$$
{ }_{p} \mathrm{~F}_{q}\left(\begin{array}{c}
\alpha_{1}, \alpha_{2}, \ldots, \alpha_{p} \\
\beta_{1}, \beta_{2}, \ldots, \beta_{q}
\end{array} \mid x\right):=\sum_{n=0}^{\infty} \frac{\left(\alpha_{1}\right)_{n}\left(\alpha_{2}\right)_{n} \cdots\left(\alpha_{p}\right)_{n}}{\left(\beta_{1}\right)_{n}\left(\beta_{2}\right)_{n} \cdots\left(\beta_{q}\right)_{n}} \cdot \frac{x^{n}}{n !}
$$

for the given non-negative integer numbers $p$ and $q$, complex parameters $\alpha_{1}, \alpha_{2}, \ldots, \alpha_{p}$, $\beta_{1}, \beta_{2}, \ldots, \beta_{q}$ and $x$ (see, e.g., [3, §2.1]), where $(z)_{0}:=1,(z)_{n}:=z(z+1)(z+2) \cdots(z+n-1)$ $(n \geq 1)$ is the Pochhammer symbol. Using series (1.1), one can represent most of elementary and special functions, as well as constants that arise in mathematics and physics (see, e.g., [19]). Hence, this class of series has many applications in the approximation theory and numerical analysis.

Evaluating a generalized hypergeometric series of the form (1.1) can be a very challenging numerical problem. First, its convergence or divergence depends mainly on the values of the numbers $p$ and $q$. Second, the variety of the parameters $\alpha_{j}, \beta_{j}, x$ leads to different types of the convergence or divergence of the series.

It is worth mentioning that the sequence transformations may be very useful numerical tools for the summation of the series given by (1.1). In the most recent edition of the book Numerical Recipes by Press et al., some classic sequence transformations are described involving summation of convergent or divergent series; see [21, §5.3].

Probably the best known class of sequence transformations are Padé approximants which deal with partial sums of the power series and transform them to a double indexed sequence of rational approximants. For further information on Padé approximants, we refer to the books by Baker [4] or by Baker and Graves-Morris [5].

\footnotetext{
*Institute of Computer Science, University of Wrocław, Poland, e-mail: rafal.nowak@cs.uni.wroc.pl, corresponding author

${ }^{\dagger}$ Institute of Computer Science, University of Wrocław, Poland, e-mail: Pawel.Wozny@ii.uni.wroc.pl

${ }^{\ddagger}$ Rev. 2355, August 30, 2016, 13:00:23Z
} 
As an alternative to the theory of Padé approximants, one can use the sequence transformations, for which the fundamentals were given by Wimp [31], and the extrapolation methods described by Bezinski and Redivo Zaglia in [7] or Sidi [24. Most of the classic algorithms were also well summarized by Weniger in the report [28] and Homeier in [11. Undoubtedly these sequence transformations have many common properties with Padé approximants, but in the case of generalized hypergeometric series they can be much more powerful tools. It is worth mentioning that evaluation of special functions with the help of convergence acceleration techniques is also considered in the book by Olver et al. [19, §3.9].

In general, the success of convergence acceleration of the series $\sum_{n=0}^{\infty} a_{n}$ very often depends on the behavior of the sequence of its partial sums $s_{n}:=\sum_{j=0}^{n-1} a_{j}$ and remainders $r_{n}:=\sum_{j=0}^{\infty} a_{n+j}$. Supposing $s_{n} \rightarrow s$, one has $s=s_{n}+r_{n}$. We say that $\left\{s_{n}\right\}$ converges linearly, if $\lim _{n \rightarrow \infty}\left(s_{n+1}-s\right) /\left(s_{n}-s\right)=: \varsigma$ and $0<|\varsigma|<1$. In the case of $\varsigma=1$, we have logarithmic convergence, which is usually the most difficult to accelerate.

If $p-q=1$ then the series (1.1) may be extremely slowly convergent and practically unusable. Therefore, the methods of convergence acceleration of such series are particularly important. For instance, in order to use any of so-called Levin-type sequence transformations, one should first provide good estimates $\omega_{n}$ of the remainders $r_{n}$; see, e.g., [11. This can be achieved with the help of recent results given by Willis in [30]. Namely, he analyzed the following asymptotic relation for the remainders of the series (1.1) with $p-q=1$ :

$$
s-s_{n}=r_{n} \sim \mu x^{n} n^{\lambda} \sum_{k=0}^{\infty} \frac{c_{k}}{n^{k}} .
$$

He derived the recurrence relation for the coefficients $c_{k}$, which gives the ability to approximate the remainders $r_{n}$ up to the desired order. Willis combined this asymptotic property with a classic technique of extrapolation and obtained a numerical algorithm of computing 2-dimensional array containing approximations of the limit of the series. Namely, the truncated estimate

$$
\omega_{n}^{(m)}:=x^{n} n^{\lambda} \sum_{k=0}^{\infty} \frac{c_{k}}{n^{k}}, \quad m \in \mathbb{N},
$$

is such that

$$
s=s_{n}+\mu \omega_{n}^{(m)}+\mathcal{O}\left(x^{n} n^{\lambda-m}\right)
$$

and thus the new approximation defined by the weighted average

$$
s_{n}^{(m)}:=\frac{\omega_{n+1}^{(m)} s_{n}-\omega_{n}^{(m)} s_{n+1}}{\omega_{n+1}^{(m)}-\omega_{n}^{(m)}}
$$

is a better approximation of the partial sum $s_{n}$ in the sense that the sequence $s_{n}^{(m)}$ converges to the limit $s$ faster than the sequence of partial sums $s_{n}$. It is worth remarking that Willis' method is very efficient at the branch point $x=1$. However, it does not seem to provide good numerical properties, if $x \approx 1$.

In this paper, we continue the analysis of $\mathscr{Q}$ transformation, introduced by us in 33 , applied to the summation of generalized hypergeometric series (1.1) with $p-q=1$. Following the notation given in 33], we consider the series

$$
\sum_{n=0}^{\infty} a_{n} \quad \text { with } \quad a_{n}:=\frac{\left(\alpha_{1}\right)_{n}\left(\alpha_{2}\right)_{n} \cdots\left(\alpha_{p}\right)_{n}}{\left(\beta_{1}\right)_{n}\left(\beta_{2}\right)_{n} \cdots\left(\beta_{p}\right)_{n}} x^{n} \quad(x \in \mathbb{C})
$$


where $\boldsymbol{\alpha}:=\left(\alpha_{1}, \alpha_{2}, \ldots, \alpha_{p}\right), \boldsymbol{\beta}:=\left(\beta_{1}, \beta_{2}, \ldots, \beta_{p}\right)$ are vectors of complex parameters. Notice that the series (1.3) corresponds to the following generalized hypergeometric series

$$
{ }_{p+1} \mathrm{~F}_{p}\left(\begin{array}{c}
\alpha_{1}, \alpha_{2}, \ldots, \alpha_{p}, 1 \\
\beta_{1}, \beta_{2}, \ldots, \beta_{p}
\end{array} \mid x\right)
$$

which is the case of the series (1.1) with $p-q=1$. Let us remark that $\mathscr{Q}$ transformation can be applied to the series (1.1) also in the case with none of the upper parameters being equal to 1 , since one can always use the following obvious relation

$$
{ }_{r} \mathrm{~F}_{s}\left(\begin{array}{c}
\alpha_{1}, \alpha_{2}, \ldots, \alpha_{r} \\
\beta_{1}, \beta_{2}, \ldots, \beta_{s}
\end{array} \mid x\right)={ }_{r+1} \mathrm{~F}_{s+1}\left(\begin{array}{c}
\alpha_{1}, \alpha_{2}, \ldots, \alpha_{r}, 1 \\
\beta_{1}, \beta_{2}, \ldots, \beta_{s}, 1
\end{array} \mid x\right) .
$$

The main purpose of this paper is to give more theoretical properties of the $\mathscr{Q}$ transformation. For a detailed comparison with other methods of convergence acceleration, we refer to [33] and [32], where the bunch of numerical examples involving many classic and recent sequence transformations, such as Aitken's iterated $\Delta^{2}$ process [2], Wynn's $\varepsilon$-algorithm [34, $t$ and $u$ variants of Levin [13] and Weniger [29] transformations, Homeier's transformations [10], method proposed by Lewanowicz and Paszkowski [14, method proposed by Čížek et al. [8] and the method of Paszkowski [20], were given; see also the Brezinski's review of convergence acceleration techniques [6].

The convergence of the series (1.3) depends on the variety of parameters $\boldsymbol{\alpha}, \boldsymbol{\beta}$ and the complex number $x$. If $|x|<1$, then the series converges absolutely. On the unit circle, the convergence is more subtle and depends on the real part of the parameter $\sigma:=1+\sum_{i=1}^{p} \alpha_{i}-$ $\sum_{i=1}^{p} \beta_{i}$. Namely, the series (1.3) converges at $x=1$, if $\Re \sigma<0$, as well as for $|x|=1(x \neq 1)$, if $\Re \sigma<1$. Many other mathematical properties of the generalized hypergeometric series ${ }_{p+1} F_{p}$ can be found in such classic sources as [1], [3], [15] or [25]. It is also worth mentioning the recent research of Miller and Paris [16, 17, 18, Rathie and Paris [22] and Kim et al. [12, where certain transformations were proposed to simplify the so-called order of generalized hypergeometric series. Several refinements of such a class of series can be also found in the very recent work of Wang [27].

In [33], the authors introduced the technique of summation of some slowly convergent series, which appeared to be very efficient also in the case of the generalized hypergeometric series. The proposed $\mathscr{Q}^{(m)}$ transformation is defined by a certain linear difference operator $\mathbb{L}_{n}^{(m)}$ in the following way:

$$
\mathscr{Q}_{n}^{(m)}:=\frac{\mathbb{L}_{n}^{(m)}\left(s_{n}\right)}{\mathbb{L}_{n}^{(m)}(1)}, \quad m \in \mathbb{N} .
$$

Here, and in the sequel, every difference operator acts upon $n$ and not upon $m$. The meaning of the linear operator $\mathbb{L}_{n}^{(m)}$ is that it annihilates a finite part of the remainder $r_{n}$. More precisely, it is required that

$$
\mathbb{L}_{n}^{(m)}\left(a_{n}+a_{n+1}+\ldots+a_{n+m-1}\right)=0 .
$$

In a general case, for arbitrary sequence $a_{n}$, the computation of the quantities $\mathscr{Q}_{n}^{(m)}$ is rather complicated and the numerical version of the algorithm is recommended; see 32. However, in the case of the series (1.3), one can compute the quantities $\mathscr{Q}_{n}^{(m)}$ in a very efficient way, i.e., using the algorithm involving certain recurrence formulas for numerators and denominators in (1.4); cf. [33, Alg. 1, Thm. 2]. 
For convenience of reference, we briefly summarize the main results from [33] in the case of the series (1.3). For a given vector $\gamma=\left(\gamma_{1}, \gamma_{2}, \ldots, \gamma_{p}\right)$, we use the following shorthand notation

$$
\langle\gamma\rangle_{n}:=\prod_{j=1}^{p}\left(\gamma_{j}\right)_{n}, \quad n \in \mathbb{N} \cup\{0\},
$$

and write $a_{n}=\langle\boldsymbol{\alpha}\rangle_{n} /\langle\boldsymbol{\beta}\rangle_{n} x^{n}$. The operators $\mathbb{L}_{n}^{(m)}$ satisfying (1.5) can be written in the form

$$
\mathbb{L}_{n}^{(m)}=\Delta^{m p}\left(\frac{\langle\boldsymbol{\beta}\rangle_{n+m-1}}{\langle\boldsymbol{\alpha}\rangle_{n} x^{n}} \mathbb{I}\right), \quad m \in \mathbb{N}
$$

see [33, Eq. (3.4)]. Here, the forward difference operator $\Delta$ and the identity operator $\mathbb{I}$ are defined according to $\Delta z_{n}:=z_{n+1}-z_{n}$ and $\mathbb{I} z_{n}:=z_{n}$, respectively; higher powers of the operator $\Delta$ are defined recursively, i.e., $\Delta^{0} z_{n}:=z_{n}$ and $\Delta^{m} z_{n}:=\Delta\left(\Delta^{m-1} z_{n}\right), m \in \mathbb{N}$. From a computational point of view, it is worth noting that operators $\mathbb{L}_{n}^{(m)}$ can be written also in the factored form $\mathbb{L}_{n}^{(m)}=\mathbb{P}_{n}^{(m)} \mathbb{P}_{n}^{(m-1)} \cdots \mathbb{P}_{n}^{(1)}$, where the operators $\mathbb{P}_{n}^{(m)}$ are defined by

$$
\begin{aligned}
\mathbb{P}_{n}^{(1)} & :=\Delta^{p}\left(\frac{\langle\boldsymbol{\beta}\rangle_{n}}{\langle\boldsymbol{\alpha}\rangle_{n}} x^{-n} \mathbb{I}\right) \\
\mathbb{P}_{n}^{(m)} & :=\sum_{j=0}^{p}\left(\begin{array}{c}
m p \\
j
\end{array}\right)\left[\Delta^{j} \prod_{j=1}^{p}\left(\beta_{j}+n+m(p+1)-j-2\right)\right] \Delta^{p-j}, \quad m \geq 2 .
\end{aligned}
$$

Thus, the quantities $\mathscr{Q}_{n}^{(m)}$ can be computed using the following recursive scheme (see [33, Alg. 1]):

$$
\begin{array}{rlrl}
N_{n}^{(0)} & :=s_{n}, & D_{n}^{(0)}:=1, & \\
N_{n}^{(m)} & :=\mathbb{P}_{n}^{(m)}\left(N_{n}^{(m-1)}\right), & D_{n}^{(m)}:=\mathbb{P}_{n}^{(m)}\left(D_{n}^{(m-1)}\right), \quad m \geq 1, \\
\mathscr{Q}_{n}^{(m)} & =\frac{N_{n}^{(m)}}{D_{n}^{(m)}} & &
\end{array}
$$

From eqs. (1.8) and (1.9), one may conclude that

$$
\mathscr{Q}_{n}^{(m)}=\mathscr{Q}_{n}^{(m)}\left(s_{n}, s_{n+1}, \ldots, s_{n+\ell(m)}\right),
$$

where $\ell(m)=m p$, which means that $\mathscr{Q}^{(m)}$ transforms the sequence $\left\{s_{n}\right\}_{n=0}^{\infty}$ to the sequence $\left\{\mathscr{Q}_{n}^{(m)}\right\}_{n=0}^{\infty}$ whose $n$-th element depends on $s_{n}, s_{n+1}, \ldots, s_{n+m p}$. Let us remark that Levin-type sequence transformations produce the double indexed quantities

$$
\mathcal{L}_{n}^{(m)}:=\mathcal{L}_{n}^{(m)}\left(\left\{\omega_{n}\right\}, s_{n}, s_{n+1}, \ldots, s_{n+\ell(m)}\right),
$$

depending both on the partial sums and on the sequence of remainder estimates $\left\{\omega_{n}\right\}$ (see, e.g., [11]), with such relationships as $\ell(m)=m, \ell(m)=m+1, \ell(m)=2 m$ or $\ell(m)=3 m$; see, e.g., [28, §2.7]. For example, the classic variants of Levin transformation have $\ell(m)=m$ and involve the following choices of remainder estimates: $\omega_{n}=a_{n}, \omega_{n}=a_{n+1}, \omega_{n}=(n+1) a_{n}$ or $\omega_{n}=\frac{a_{n} a_{n+1}}{a_{n}-a_{n+1}}$; see the paper of Levin [13] and work of Smith and Ford [26]. The advantage of $\mathscr{Q}^{(m)}$ transformation is that the information about remainder estimates $\omega_{n}$ is a priori hidden in the analytic form of the operators $\mathbb{P}_{n}^{(m)}$, given by the explicit formulas (1.8). It should be remarked that the operators (1.8) seem to provide the transformation $\mathscr{Q}^{(m)}$ which is a very 
powerful numerical tool for the summation of the generalized hypergeometric series (1.1) with $p-q=1$.

Some theoretical properties of $\mathscr{Q}^{(m)}$ transformation were also given in [33]. In the case of the series (1.3), we can summarize them as follows.

If $p=1$, then $\mathscr{Q}^{(m)}$ transformation is equivalent to Wynn's $\varepsilon$ algorithm [34] in the sense that $\mathscr{Q}_{n}^{(m)}=\varepsilon_{2 m}^{(n)}$, which follows from the general property given in [33, §2.3]. It is worth mentioning that the explicit formula for $\varepsilon_{2 m}^{(n)}$ in the case of ${ }_{2} \mathrm{~F}_{1}(\alpha, 1 ; \beta \mid x)$ has already been given by Sidi in [23, Ex. 2] (see also [24, §17.3]). The mentioned relation between $\mathscr{Q}^{(m)}$ and $\varepsilon$ transformations does not hold for $p>1$.

Supposing that the series (1.3) is convergent, we also know that $\mathscr{Q}^{(m)}$ transformation is regular for all $m \in \mathbb{N}$, if $x \neq 1$, i.e.,

$$
\lim _{n \rightarrow \infty} \mathscr{Q}_{n}^{(m)}\left(s_{n}\right)={ }_{p+1} \mathrm{~F}_{p}\left(\begin{array}{c}
\alpha_{1}, \alpha_{2}, \ldots, \alpha_{p}, 1 \\
\beta_{1}, \beta_{2}, \ldots, \beta_{p}
\end{array} \mid x\right)
$$

see [33, Thm. 5]. What is more, it possesses the following asymptotic behavior:

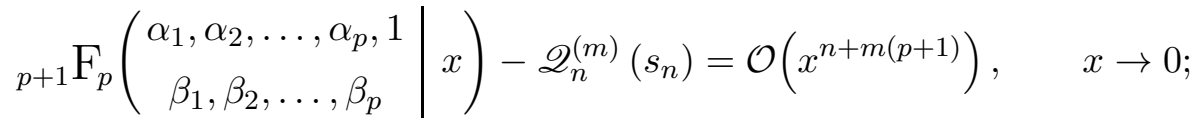

see [33, Thm. 6].

Moreover, a lot of numerical tests show that the sequence $\mathscr{Q}_{n}^{(m)}$ not only converges to the limit of the series but also converges much faster for bigger and bigger values of $m$.

The paper is organized as follows. In Section 2, we give some new properties of $\mathscr{Q}^{(m)}$ transformation in the case of the series (1.3) including the main result, which is the convergence acceleration theorem. Later, in Section 3, we give some numerical examples and, in Section 4, discuss further problems concerning theoretical properties of $\mathscr{Q}^{(m)}$ transformation.

\section{Main result}

Let us consider the transformation $\mathscr{Q}^{(m)}$, defined in (1.4), in the case of the series (1.3). Following [33], we define the functions $\lambda_{j}^{(m)}(n) \equiv \lambda_{j}^{(m)}(n, p, x, \boldsymbol{\alpha}, \boldsymbol{\beta})$ by

$$
\lambda_{j}^{(m)}(n):=\frac{\langle\boldsymbol{\alpha}\rangle_{n+m p} x^{n+m p}}{\langle\boldsymbol{\beta}\rangle_{n+m-1}}\left[(-1)^{m p-j}\left(\begin{array}{c}
m p \\
j
\end{array}\right) \frac{\langle\boldsymbol{\beta}\rangle_{n+j+m-1}}{\langle\boldsymbol{\alpha}\rangle_{n+j} x^{n+j}}\right], \quad j=0,1, \ldots, m p,
$$

and thus, using (1.7), we can write the transformation $\mathscr{Q}^{(m)}$ as follows:

$$
\mathscr{Q}_{n}^{(m)}=\frac{\mathbb{L}^{(m)}\left(s_{n}\right)}{\mathbb{L}^{(m)}(1)}=\frac{\sum_{j=0}^{m p} \lambda_{j}^{(m)}(n) s_{n+j}}{\sum_{j=0}^{m p} \lambda_{j}^{(m)}(n)} .
$$

Since

$$
\lambda_{j}^{(m)}(n)=\left(\begin{array}{c}
m p \\
j
\end{array}\right)(-x)^{m p-j} \prod_{i=1}^{p}\left[\left(\alpha_{i}+n+j\right)_{m p-j}\left(\beta_{i}+n+m-1\right)_{j}\right]
$$

(see [33, Eq. (3.11)]), the quantity $\mathscr{Q}_{n}^{(m)}$ is a linear combination of the quantities $s_{n}, s_{n+1}, \ldots$, $s_{n+m p}$ with coefficients $\lambda_{j}^{(m)}(n)$ being polynomials of degree $m p^{2}$ in $n$. In the lemma below, 
we express the element $\mathscr{Q}_{n}^{(m)}$ in terms of $s_{n}$ and $a_{n}, a_{n+1}, \ldots, a_{n+m p-1}$. In the sequel, we use the following polynomials in $n$ :

$$
M^{(m)}(n) \equiv M_{0}^{(m)}(n), \quad M_{k}^{(m)}(n):=\sum_{j=k}^{m p} \lambda_{j}^{(m)}(n), \quad k=0,1, \ldots, m p .
$$

Lemma 2.1. The quantity $\mathscr{Q}_{n}^{(m)}$ can be written as the following linear combination involving the partial sum $s_{n}$ and the terms $a_{n}, a_{n+1}, \ldots, a_{n+m p-1}$ :

$$
\mathscr{Q}_{n}^{(m)}=s_{n}+\sum_{k=0}^{m p-1} \frac{M_{k+1}^{(m)}(n)}{M^{(m)}(n)} a_{n+k}, \quad m, n \in \mathbb{N} .
$$

Proof. First, let us observe that $\mathscr{Q}_{n}^{(m)}=\sum_{j=0}^{m p} \lambda_{j}^{(m)}(n) s_{n+j} / M^{(m)}(n)$. Second, we have

$$
\begin{aligned}
\mathscr{Q}_{n}^{(m)}=\frac{\sum_{j=0}^{m p} \lambda_{j}^{(m)}(n)\left(s_{n}+a_{n}+a_{n+1}+\ldots+a_{n+j-1}\right)}{M^{(m)}(n)} & \\
= & \frac{\sum_{j=0}^{m p} \lambda_{j}^{(m)}(n) s_{n}+\sum_{k=0}^{m p-1}\left(\sum_{j=k+1}^{m p} \lambda_{j}^{(m)}(n)\right) a_{n+k}}{M^{(m)}(n)}=s_{n}+\sum_{k=0}^{m p-1} \frac{M_{k+1}^{(m)}(n)}{M^{(m)}(n)} a_{n+k} .
\end{aligned}
$$

Now, we are going to give some theoretical results about the convergence acceleration performed by $\mathscr{Q}$ transformation. The first theorem gives the necessary and sufficient condition for the convergence acceleration. Next, we show that this condition holds under a certain assumption which is discussed later - in Subsection 2.1. The statement concerning the convergence acceleration of the linearly convergent series (1.3) is given thereafter. We also analyze the convergence at the points $x= \pm 1$.

Theorem 2.2. Consider the series (1.3) with partials sums $s_{n}$ converging to $s$. The transformation $\mathscr{Q}^{(m)}$ accelerates the convergence of $\left\{s_{n}\right\}$, i.e.,

$$
\lim _{n \rightarrow \infty} \frac{\mathscr{Q}_{n}^{(m)}-s}{s_{n}-s}=0, \quad m \in \mathbb{N},
$$

if and only if

$$
\lim _{n \rightarrow \infty} \sum_{k=0}^{m p-1} \frac{M_{k+1}^{(m)}(n)}{M^{(m)}(n)} \cdot \frac{a_{n+k}}{r_{n}}=1,
$$

where $M_{k}(n)$ and $M(n)$ are defined by (2.3) .

Proof. The theorem follows immediately from Lemma 2.1, since

$$
\lim _{n \rightarrow \infty} \frac{\mathscr{Q}_{n}^{(m)}-s}{s_{n}-s}=1-\lim _{n \rightarrow \infty}\left(\frac{1}{r_{n}} \sum_{k=0}^{m p-1} \frac{M_{k+1}^{(m)}(n)}{M^{(m)}(n)} a_{n+k}\right) .
$$


In the next theorem, we show that the condition (2.6) is fulfilled for a certain class of convergent hypergeometric series (1.3). Let us remark that the further properties hold upon the following assumption.

Assumption 2.3. We assume that the remainders of the series (1.3) satisfy

$$
\lim _{n \rightarrow \infty} \frac{r_{n+1}}{r_{n}}=x .
$$

Theorem 2.4. If Assumption 2.3 holds and $x \neq 1$, then the condition (2.6) is satisfied for all $m \in \mathbb{N}$, and thus the transformation $\mathscr{Q}^{(m)}$ accelerates the convergence of $\left\{s_{n}\right\}$.

Proof. Let $c_{j}^{(m)}$ be the coefficient for the term $n^{m p^{2}}$ in $\lambda_{j}^{(m)}(n)$, i.e.,

$$
c_{j}^{(m)}:=\left(\begin{array}{c}
m p \\
j
\end{array}\right)(-x)^{m p-j}, \quad j=0,1, \ldots, m p
$$

cf. (2.2). Using binomial theorem, one can check that the following two relationships hold:

$$
\sum_{j=0}^{m p} c_{j}^{(m)}=(1-x)^{m p}, \quad \sum_{j=0}^{m p} c_{j}^{(m)} x^{j}=0 .
$$

Therefore, if $x \neq 1$, the polynomial $M^{(m)}(n)$, given by (2.3), is exactly of degree $m p^{2}$. This yields

$$
\lim _{n \rightarrow \infty} \frac{M_{k+1}^{(m)}(n)}{M^{(m)}(n)}=\sum_{j=k+1}^{m p} c_{j}^{(m)} / \sum_{j=0}^{m p} c_{j}^{(m)}=(1-x)^{-m p} \sum_{j=k+1}^{m p} c_{j}^{(m)} .
$$

Using the fact that $a_{n+k}=r_{n+k}-r_{n+k+1}$ and Assumption 2.3, we obtain

$$
\begin{aligned}
\lim _{n \rightarrow \infty} \sum_{k=0}^{m p-1} \frac{M_{k+1}^{(m)}(n)}{M^{(m)}(n)} \cdot \frac{a_{n+k}}{r_{n}}=(1-x)^{-m p} \sum_{k=0}^{m p-1} \sum_{j=k+1}^{m p} c_{j}^{(m)}\left(x^{k}-x^{k+1}\right) & =(1-x)^{-m p}\left(\sum_{j=0}^{m p} c_{j}^{(m)}-\sum_{j=0}^{m p} c_{j}^{(m)} x^{j}\right) .
\end{aligned}
$$

Now, the result follows from (2.7).

\subsection{Some comments}

Let us note that Assumption 2.3 holds for each series (1.3) with $|x|<1$, which implies the linear convergence. It follows directly from [31, Thm. 1, p. 6] providing that

$$
x=\lim _{n \rightarrow \infty} \frac{a_{n+1}}{a_{n}}=\lim _{n \rightarrow \infty} \frac{r_{n+1}}{r_{n}},
$$

if $|x|<1$. This yields the theoretical explanation of convergence acceleration performed by $\mathscr{Q}^{(m)}$ transformation in the case of linearly convergent series (1.3).

Assumption 2.3 is also satisfied if $x=1$ and all the terms $a_{n}$ are real and have the same sign; see [9, Thm. 2, p. 26]. In this case, the series (1.3) converges logarithmically. It is quite remarkable that the transformation $\mathscr{Q}^{(m)}$ seems to be very efficient also in such a case. However, we cannot use Theorem 2.4 for $x=1$ in order to justify that $\mathscr{Q}^{(m)}$ transformation leads to an acceleration of the convergence. 
Let us remark that Assumption 2.3 holds also for a certain class of the series (1.3) with $x=-1$. Namely, from [9, Thm. 3, p. 26], we obtain that it is satisfied, if $\langle\boldsymbol{\alpha}\rangle_{n} /\langle\boldsymbol{\beta}\rangle_{n}$ decreases monotonically to zero and

$$
\lim _{n \rightarrow \infty} \frac{1+t_{n+1}}{1+t_{n}}=1
$$

where $t_{n}:=a_{n+1} / a_{n}$.

In the next section, we give some comments and numerical examples showing the consequences of the given theorems.

\section{$3 \quad$ Experiments}

Since the main purpose of this paper is to give the theoretical properties of the $\mathscr{Q}^{(m)}$ transformation, we refer to [33] and [32], for the numerical examples displaying, among other things, the detailed comparison with many classic methods of convergence acceleration. However, we would like to give some new examples in order to depict the conclusions of the theory given in Section 2 ,

We use $\mathscr{Q}^{(m)}$ transformation in order to approximate the sum of the generalized hypergeometric series ${ }_{3} \mathrm{~F}_{2}\left(\alpha_{1}, \alpha_{2}, 1 ; \beta_{1}, \beta_{2} \mid x\right)$. Let us remark that one can compute the array of quantities $\mathscr{Q}_{n}^{(m)}$ using the recurrence formulas (1.9) and replacing the operators $\mathbb{P}_{n}^{(m)}$ with the equivalent ones:

$$
\begin{aligned}
& \mathbb{P}_{n}^{(m)}:=x^{2}\left(n+2 m+\alpha_{1}-2\right)_{2}\left(n+2 m+\alpha_{2}-2\right)_{2} \mathbb{I} \\
& -2 x\left(n+2 m+\alpha_{1}-1\right)\left(n+2 m+\alpha_{2}-1\right)\left[\left(n+2 m+\beta_{1}-2\right)\left(n+2 m+\beta_{2}-2\right)-m^{2}+m\right] \mathbb{E} \\
& \quad+\left(n+m+\beta_{1}-1\right)\left(n+m+\beta_{2}-1\right)\left(n+3 m+\beta_{1}-2\right)\left(n+3 m+\beta_{2}-2\right) \mathbb{E}^{2}
\end{aligned}
$$

(cf. [33, Eq. (3.8)]), where the forward shift operators $\mathbb{E}$ and $\mathbb{E}^{2}$ are defined according to $\mathbb{E} z_{n}:=$ $z_{n+1}$, and $\mathbb{E}^{2} z_{n}:=z_{n+2}$.

In all the examples, we start with the column $\mathscr{Q}_{n}^{(0)}$ containing some finite number of partial sums of the series. Next, we use recurrence formulas (1.9) and obtain the triangular array of the quantities $\mathscr{Q}_{n}^{(m)}$.

In the first example, we consider the alternating series (1.3) with $x=-1$. In this case, one can use Theorem 2.4 in order to explain the convergence acceleration performed by the $\mathscr{Q}$ transformation. Indeed, we obtain highly accurate approximations in the triangular array $\mathscr{Q}_{n}^{(m)}$.

In the second example, we consider the linearly convergent series (1.3). In order to obtain a very slow convergence, we take $x \approx 1$. Again, as a consequence of Theorem 2.4 , the transformation $\mathscr{Q}$ provides a quite good approximation of the limit of the series.

In the last example, we consider the series (1.3) with $x=1$, which means the logarithmic convergence. One can observe that the $\mathscr{Q}$ transformation is very efficient, although this good performance cannot be justified by Theorem 2.4.

All the numerical experiments were made in Maple ${ }^{\mathrm{TM}} 14$ system, using floating point arithmetic with 32 decimal digits precision. Consequently, as in [33], we measure the accuracy of the approximation $z$ of the sum $s \neq 0$ by the number of exact significant decimal digits, in the following sense:

$$
\operatorname{acc}(z):=-\log _{10}\left|\frac{z}{s}-1\right| .
$$


Example 3.1. Let us consider the following expansion of the integral involving the square root function:

$$
\frac{1}{z} \int_{0}^{z} \sqrt{1+y^{\alpha}} \mathrm{d} y={ }_{2} \mathrm{~F}_{1}\left(\begin{array}{c}
\frac{1}{\alpha},-\frac{1}{2} \\
1+\frac{1}{\alpha}
\end{array} \mid-z^{\alpha}\right) \quad(\alpha>0,0<z \leq 1) .
$$

Since the first parameter of the hypergeometric series is not equal to 1 , we consider the convergence acceleration of the series ${ }_{3} \mathrm{~F}_{2}\left(1 / \alpha,-1 / 2,1 ; 1+1 / \alpha, 1 \mid-z^{\alpha}\right)$. We put $\alpha=1 / 3$ and $z=1$. Thus, we obtain an alternating series of the form (1.3) with

$$
a_{n}=\frac{3\left(-\frac{1}{2}\right)_{n}}{(n+3) n !} x^{n}, \quad x=-1,
$$

converging to $(44 \sqrt{2}-16) / 35 \approx 1.3207256213$. The convergence is quite slow since straightforward computation gives (underline depicts the correct digits):

$$
s_{10}=\underline{1.32} 19178336, \quad s_{100}=\underline{1.32072} 97959, \quad s_{1000}=\underline{1.3207256} 346, \quad s_{10000}=\underline{1.3207256213} .
$$

This yields the following number of exact significant decimal digits of the limit of the series:

$$
\operatorname{acc}\left(s_{10}\right)=3.0, \quad \operatorname{acc}\left(s_{100}\right)=5.5, \quad \operatorname{acc}\left(s_{1000}\right)=8.0, \quad \operatorname{acc}\left(s_{10000}\right)=10.5 .
$$

One can check that $t_{n}:=a_{n+1} / a_{n}=-[(2 n-1)(n+3)] /[(2 n+2)(n+4)]$, and thus the equation (2.8) holds. Since the fraction in (3.3) decreases monotonically to zero, we conclude that Assumption 2.3 is satisfied; cf. [9, Thm. 3, p. 26]. From Theorem 2.4, we obtain that $\mathscr{Q}^{(m)}$ transformation accelerates the convergence of the considered series. Indeed, the accuracy of the quantities $\mathscr{Q}_{n}^{(m)}$ increases for bigger and bigger values of $m$; see Table1. It is worth noting that the quantity $\mathscr{Q}_{1}^{(7)}$ gives about 21 digits of accuracy, while the partial sums $s_{1}, s_{2}, \ldots, s_{15}$, that it depends on (see Lemma 2.11), give less than 4 digits.

Table 1: Values of $\operatorname{acc}\left(\mathscr{Q}_{n}^{(m)}\right)$ for the hypergeometric series (3.2) with $\alpha=1 / 3$ and $z=1$.

\begin{tabular}{c|rcrrrrrr}
$n \backslash m$ & \multicolumn{1}{|c}{1} & 1 & \multicolumn{1}{c}{2} & \multicolumn{1}{c}{3} & \multicolumn{1}{c}{4} & \multicolumn{1}{c}{5} & \multicolumn{1}{c}{6} & 7 \\
\hline 1 & 0.6 & 3.1 & 6.1 & 9.5 & 12.6 & 15.9 & 19.3 & 21.7 \\
2 & 1.4 & 3.8 & 6.9 & 10.3 & 13.4 & 16.9 & 19.9 & \\
3 & 1.8 & 4.4 & 7.6 & 11.0 & 14.2 & 17.9 & 20.6 & \\
4 & 2.1 & 4.9 & 8.2 & 11.6 & 14.9 & 18.9 & & \\
5 & 2.3 & 5.3 & 8.7 & 12.1 & 15.6 & 20.6 & & \\
6 & 2.5 & 5.6 & 9.2 & 12.7 & 16.2 & & & \\
7 & 2.7 & 5.9 & 9.6 & 13.1 & 16.7 & & & \\
8 & 2.8 & 6.2 & 10.0 & 13.6 & & & & \\
9 & 2.9 & 6.4 & 10.4 & 14.0 & & & & \\
10 & 3.0 & 6.6 & 10.7 & & & & & \\
11 & 3.1 & 6.8 & 11.0 & & & & & \\
12 & 3.2 & 7.0 & & & & & & \\
13 & 3.3 & 7.2 & & & & & & \\
14 & 3.4 & & & & & & & \\
15 & 3.5 & & & & & & &
\end{tabular}


Example 3.2. Let us consider the linearly convergent series

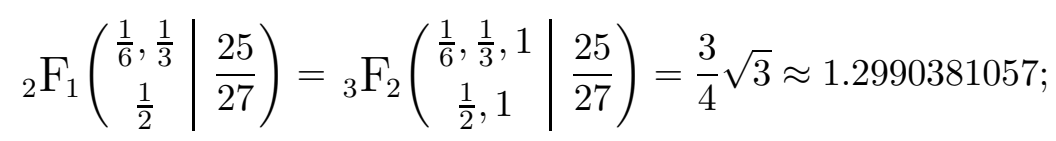

see [35]. The straightforward computation yields

$$
s_{10}=\underline{1.2} 573432291, \quad s_{100}=\underline{1.29903} 15516, \quad s_{200}=\underline{1.29903810} 41 .
$$

This means the following accuracies:

$$
\operatorname{acc}\left(s_{10}\right)=1.5, \quad \operatorname{acc}\left(s_{100}\right)=5.3, \quad \operatorname{acc}\left(s_{200}\right)=8.9 .
$$

However, using only partial sums $s_{1}, s_{2}, \ldots, s_{25}$, one can compute the triangular array of the elements $\mathscr{Q}_{n}^{(m)}$ for $1 \leq n+2 m \leq 25,0 \leq m \leq 12$. For instance, we have

$$
\mathscr{Q}_{1}^{(10)}=\underline{1.29903810} 82, \quad \mathscr{Q}_{1}^{(11)}=\underline{1.2990381060}, \quad \mathscr{Q}_{1}^{(12)}=\underline{1.2990381057},
$$

which yields the following accuracies:

$$
\operatorname{acc}\left(\mathscr{Q}_{1}^{(10)}\right)=7.0, \quad \operatorname{acc}\left(\mathscr{Q}_{1}^{(11)}\right)=7.9, \quad \operatorname{acc}\left(\mathscr{Q}_{1}^{(12)}\right)=8.7 .
$$

The following is worth remarking: $1^{\circ}$ for the fixed subscript $n$, the quantities $\mathscr{Q}_{n}^{(m)}$ (for consecutive values of $m$ ) are of better and better accuracy (namely, every next element has, more or less, one exact decimal digit more); $2^{\circ}$ since Assumption 2.3 is satisfied, this is in agreement with Theorem 2.4, providing that

$$
\lim _{n \rightarrow \infty} \frac{\mathscr{Q}_{n}^{(m)}-s}{s_{n}-s}=0
$$

which means that each column of the array $\mathscr{Q}_{n}^{(m)}$ converges to $s$ faster than the sequence of partial sums $s_{n} ; 3^{\circ}$ the accuracy of the quantities $\mathscr{Q}_{n}^{(m)}$ shows the faster and faster convergence for consecutive columns of the table.

Example 3.3. Let us consider the complex vectors of parameters: $\boldsymbol{\alpha}=(1.7+2.5 i, 1.5+2.0 i)$, $\boldsymbol{\beta}=(1.3-3.0 i, 3.2-4.0 i)$. The hypergeometric series

$$
{ }_{3} \mathrm{~F}_{2}\left(\alpha_{1}, \alpha_{2}, 1 ; \beta_{1}, \beta_{2} \mid 1\right) \approx 0.7808031959823745-0.2060305207425406 i
$$

is extremely slowly convergent. Indeed, the partial sums $s_{n}$ for $n=10^{3}, 10^{5}, 10^{6}$ give accuracy of about 2.3, 2.9 and 3.2 exact significant decimal digits, respectively. In contrast, using only partial sums $s_{1}, s_{2}, \ldots, s_{15}$, the transformation $\mathscr{Q}$ gives the quantities $\mathscr{Q}_{n}^{(m)}$ being very good approximations of the limit; see Table 2, The reason for this is that the sufficient condition in Theorem 2.2 is arguably satisfied. Indeed, the straightforward computation yields that numerical values of

$$
\sum_{k=0}^{m p-1} \frac{M_{k+1}^{(m)}(n)}{M^{(m)}(n)} \cdot \frac{a_{n+k}}{r_{n}}
$$

approach 1.0 when $n \rightarrow \infty$. Moreover, these values are closer and closer to 1.0 for bigger and bigger values of $m$. We believe this explains the convergence acceleration performed by $\mathscr{Q}$ transformation; cf. Table 2 , 
Table 2: Values of $\operatorname{acc}\left(\mathscr{Q}_{n}^{(m)}\right)$ for the hypergeometric series in Example 3.3.

\begin{tabular}{c|cccccccc}
$n \backslash m$ & 0 & 1 & 2 & 3 & \multicolumn{1}{c}{4} & \multicolumn{1}{c}{5} & \multicolumn{1}{c}{6} & 7 \\
\hline 1 & 0.4 & 2.6 & 4.6 & 6.6 & 8.5 & 10.3 & 12.2 & 14.1 \\
2 & 0.7 & 3.0 & 5.1 & 7.0 & 9.0 & 10.9 & 12.7 & \\
3 & 0.9 & 3.4 & 5.5 & 7.5 & 9.4 & 11.3 & 13.2 & \\
4 & 1.1 & 3.6 & 5.8 & 7.8 & 9.8 & 11.7 & & \\
5 & 1.2 & 3.9 & 6.1 & 8.2 & 10.2 & 12.1 & & \\
6 & 1.3 & 4.0 & 6.3 & 8.5 & 10.5 & & & \\
7 & 1.3 & 4.2 & 6.5 & 8.7 & 10.8 & & & \\
8 & 1.4 & 4.3 & 6.7 & 9.0 & & & & \\
9 & 1.4 & 4.4 & 6.9 & 9.2 & & & & \\
10 & 1.5 & 4.5 & 7.1 & & & & & \\
11 & 1.5 & 4.6 & 7.2 & & & & & \\
12 & 1.5 & 4.7 & & & & & & \\
13 & 1.5 & 4.8 & & & & & & \\
14 & 1.6 & & & & & & & \\
15 & 1.6 & & & & & & &
\end{tabular}

\section{Further problems}

Let us remark that the statement in Theorem 2.4 does not consider the case of $x=1$ in the series (1.3). This case leads to the logarithmic convergence which is usually the most difficult to sum. Although we cannot use Theorem 2.4 in order to prove the convergence acceleration for the $\mathscr{Q}$ transformation, one can always try to check if condition (2.6) is satisfied. This is exactly what was depicted in Example 3.3. We strongly believe that the condition (2.6) is fulfilled for all the classes of logarithmically convergent series satisfying Assumption 2.3. However, we do not know how to prove it in a general way. Let us remark that in this case, the polynomials $M^{(m)}(n)$, given by (2.3), are no longer of the degree $m p^{2}$; cf. (2.7). Therefore, the proof of Theorem 2.4 needs to be different in such a case. However, one can try to check if the condition (2.6) is satisfied, provided that the terms and remainders of the series (1.3) have, at least formally, the following asymptotic representation:

$$
\frac{a_{n+1}}{a_{n}} \sim 1+\frac{b_{1}}{n}+\frac{b_{2}}{n^{2}}+\ldots, \quad \frac{r_{n+1}}{r_{n}} \sim 1+\frac{d_{1}}{n}+\frac{d_{2}}{n^{2}}+\ldots
$$

Hence, one can check that

$$
\begin{aligned}
b_{1} & =\sum_{j=1}^{p} \alpha_{j}-\sum_{j=1}^{p} \beta_{j}, & b_{2} & =\frac{1}{2}\left(b_{1}^{2}-\sum_{j=1}^{p} \alpha_{j}^{2}+\sum_{j=1}^{p} \beta_{j}^{2}\right), \\
d_{1} & =b_{1}+1, & d_{2} & =\left(b_{1}^{2}+b_{1} b_{2}+b_{1}+b_{2}\right) / b_{1} .
\end{aligned}
$$

Thus, using the fact that

$$
\frac{a_{n+k}}{r_{n}}=\frac{r_{n+1}}{r_{n}} \frac{r_{n+2}}{r_{n+1}} \cdots \frac{r_{n+k}}{r_{n+k-1}}\left(1-\frac{r_{n+k+1}}{r_{n+k}}\right),
$$

one can obtain, after some algebra, that

$$
\frac{1}{r_{n}} \sum_{k=0}^{m p-1} \frac{M_{k+1}^{(m)}(n)}{M^{(m)}(n)} a_{n+k} \sim 1+\frac{\pi_{2}}{n^{2}}+\frac{\pi_{3}}{n^{3}}+\ldots
$$


This compared with condition (2.6) in Theorem 2.4 explains the acceleration of the convergence obtained in Example 3.3. But still the statement in Theorem 2.2 is an open problem in the case of a general form of the series (1.3) with $x=1$.

Maybe even more interesting is to explain why we observe that the quantities $\mathscr{Q}_{n}^{(m)}$ give better and better approximation of the limit of the series, for bigger and bigger values of $m$; cf. Tables 1 and 2. We believe that the main reason is the relationship (1.5) satisfied by the operators $\mathbb{L}_{n}^{(m)}$ defining $\mathscr{Q}^{(m)}$ transformation. However, the proof of

$$
\lim _{n \rightarrow \infty} \frac{\mathscr{Q}_{n}^{(m+1)}-s}{\mathscr{Q}_{n}^{(m)}-s}=0, \quad m \in \mathbb{N},
$$

seems to be very difficult not only in the case of logarithmic convergence, but also for a linearly convergent series (1.3). We leave it for the further research.

\section{Acknowledgements}

The authors would like to thank the anonymous referees for valuable remarks and suggestions, as well as for pointing out many interesting possibilities of future research in the area of series acceleration. We would also like to express our gratitude to Prof. E. J. Weniger for his interesting comments on our previous research and for stimulating us to further work during the conference Approximation and extrapolation of convergent and divergent sequences and series (Luminy, 2009).

\section{References}

[1] M. Abramowitz and I. A. Stegun. Handbook of Mathematical Functions. National Bureau of Standards, Washington, D.C., 10th printing edition, 1972.

[2] A. C. Aitken. On Bernoulli's numerical solution of algebraic equations. Proceedings Royal Soc. Edinburgh, 46:289-305, 1926.

[3] G. E. Andrews, R. Askey, and R. Roy. Special functions. Cambridge University Press, Cambridge, 1999.

[4] G. A. Baker, Jr. Essentials of Padé approximants. Academic Press, New York, 1975.

[5] G. A. Baker, Jr and P. Graves-Morris. Padé approximants. Part I: Basic theory. Part II: Extensions and applications. Addison-Wesley, Reading, Massachusetts, 1981.

[6] C. Brezinski. Convergence acceleration during the 20th century. J. Comput. Appl. Math., 122(1-2):1-21, 2000.

[7] C. Brezinski and M. Redivo Zaglia. Extrapolation Methods: Theory and Practice, volume 2 of Studies in Computational Mathematics. North-Holland, 1991.

[8] J. Čížek, J. Zamastil, and L. Skála. New summation technique for rapidly divergent perturbation series. Hydrogen atom in magnetic field. J. Math. Phys., 44(3):962-968, Mar. 2003 .

[9] W. D. Clark, H. L. Gray, and J. E. Adams. A note on the T-transformation of Lubkin. J. Res. Natl. Bur. Stand., 73B:25-29, 1969. 
[10] H. H. H. Homeier. A hierarchically consistent, iterative sequence transformation. Numer. Algorithms, 8(1):47-81, 1994.

[11] H. H. H. Homeier. Scalar Levin-type sequence transformations. In C. Brezinski, editor, Numerical Analysis 2000, Vol. 2: Interpolation and Extrapolation, pages 81-147, 2000.

[12] Y. S. Kim, A. K. Rathie, and R. B. Paris. On two Thomae-type transformations for hypergeometric series with integral parameter differences. Math. Commun., 19(1):111$118,2014$.

[13] D. Levin. Development of non-linear transformations for improving convergence of sequences. Int. J. Comput. Math., 3:371-388, 1973.

[14] S. Lewanowicz and S. Paszkowski. An analytic method for convergence acceleration of certain hypergeometric series. Math. Comput., 64(210):691-713, 1995.

[15] W. Magnus, F. Oberhettinger, and R. P. Soni. Formulas and theorems for the special functions of mathematical physics. Springer-Verlag, New York, 1966.

[16] A. R. Miller and R. B. Paris. Certain transformations and summations for generalized hypergeometric series with integral parameter differences. Integr. Transf. Spec. F., 22(13):67-77, 2011.

[17] A. R. Miller and R. B. Paris. On a result related to transformations and summations of generalized hypergeometric series. Math. Commun., 17(1):205-210, 2012.

[18] A. R. Miller and R. B. Paris. Transformation formulas for the generalized hypergeometric function with integral parameter differences. Rocky Mt. J. Math., 43(1):291-327, 022013.

[19] F. W. J. Olver, D. W. Lozier, R. F. Boisvert, and C. W. Clark, editors. NIST Handbook of Mathematical Functions. Cambridge University Press, New York, 2010.

[20] S. Paszkowski. Convergence acceleration of orthogonal series. Numer. Algorithms, 47(1):35$62,2008$.

[21] W. H. Press, S. A. Teukolsky, W. T. Vetterling, and B. P. Flannery. Numerical Recipes 3rd Edition: The Art of Scientific Computing. Cambridge University Press, New York, NY, USA, 2007.

[22] A. Rathie and R. Paris. Extension of some classical summation theorems for the generalized hypergeometric series with integral parameter differences. J. Class. Anal., 3:109-127, 2013.

[23] A. Sidi. A new method for deriving Padé approximants for some hypergeometric functions. J. Comput. Appl. Math., 7:37-40, 1981.

[24] A. Sidi. Practical Extrapolation Methods - Theory and Applications, volume 10 of Cambridge monographs on applied and computational mathematics. Cambridge University Press, 2003.

[25] L. J. Slater. Generalized Hypergeometric Functions. Cambridge University Press, Cambridge, 1966.

[26] D. A. Smith and W. F. Ford. Acceleration of linear and logarithmic convergence. SIAM J. Numer. Anal., 16:223-240, 1979. 
[27] M.-K. Wang, Y.-M. Chu, and Y.-Q. Song. Asymptotical formulas for gaussian and generalized hypergeometric functions. Appl. Math. Comput., 276(C):44-60, 2016.

[28] E. J. Weniger. Nonlinear sequence transformations for the acceleration of convergence and the summation of divergent series. Comput. Phys. Rep., 10:189-371, 1989.

[29] E. J. Weniger. Interpolation between sequence transformations. Numer. Algorithms, 3(14):477-486, 1992.

[30] J. Willis. Acceleration of generalized hypergeometric functions through precise remainder asymptotics. Numer. Algorithms, 59(3):447-485, 2012.

[31] J. Wimp. Sequence transformations and their applications, volume 154 of Mathematics in Science and Engineering. Academic Press, New York - London, 1981.

[32] P. Woźny. Efficient algorithm for summation of some slowly convergent series. Appl. Numer. Math., 60(12):1442-1453, 2010.

[33] P. Woźny and R. Nowak. Method of summation of some slowly convergent series. Appl. Math. Comput., 215(4):1622-1645, 2009.

[34] P. Wynn. On a device for computing the $e_{m}\left(S_{n}\right)$ transformation. Math. Tables Aids Comput., 10:91-96, 1956.

[35] I. J. Zucker and G. S. Joyce. Special values of the hypergeometric series II. Math. Proc. Cambridge, 131:309-319, 92001. 